\title{
Prevalence and Associated Factors of Dry Eye among Glaucoma Patients at KCMC Eye Department
}

\author{
Mário J. Monjane, William Makupa ${ }^{1,2,3}$ \\ ${ }^{1}$ Kilimanjaro Christian Medical University College (KCMUCO), Moshi, Tanzania \\ ${ }^{2}$ Kilimanjaro Christian Medical Center Hospital, Moshi, Tanzania \\ ${ }^{3}$ Department of Ophthalmology, Moshi, Tanzania \\ Email: mariomonjane@gmail.com,makupauw@yahoo.com
}

How to cite this paper: Monjane, M.J. and Makupa, W. (2020) Prevalence and Associated Factors of Dry Eye among Glaucoma Patients at KCMC Eye Department. Open Journal of Ophthalmology, 10, 154-163. https://doi.org/10.4236/ojoph.2020.102017

Received: February 21, 2020

Accepted: May 12, 2020

Published: May 15, 2020

Copyright ( 2020 by author(s) and Scientific Research Publishing Inc. This work is licensed under the Creative Commons Attribution International License (CC BY 4.0).

http://creativecommons.org/licenses/by/4.0/

\section{(c) (i) Open Access}

\begin{abstract}
Propose: Dry eye is a common disease among glaucoma patient undergoing on topical medication for lowering intraocular pressure. In this study we determine the prevalence and risk factors of dry eye among glaucoma patients. Methodology: We conducted a prospective cross-sectional study at Kilimanjaro Christian Medical Center (KCMC), Ophthalmology Department, over a period of 8 months, from October 2018 to June of 2019. Purposive sampling technique was used, and patients with POAG aged 18 years and above were consecutively selected. Socio-demographic and clinical data were collected. The main outcome variable was dry eye. The data were summarized using SPSS version 23 and analyzed using the STATA software version 13. Results: A total of 325 patients were enrolled into the study; $56.6 \%$ were male and $43.4 \%$ were female. The mean age was 66.1 years (SD11.7). We found that the prevalence of dry eye using OSDI questionnaire was 79.7\%; among them evaporative dry eye was found in $36.9 \%$ and aqueous deficiency in $43.4 \%$; $87.1 \%$ had abnormal TBUT; abnormal ocular surface staining was found in 64.3\%; Schirmer test was abnormal in $43.4 \%$ and $36.9 \%$ had meibomian gland dysfunction. The severity of dry eyes among those using OSDI questionnaire is: mild $22.1 \%$, moderate $16.0 \%$ and severe $61.9 \%$. Factors such as use of two or more medication ( $\mathrm{AOR}=2.55,95 \% \mathrm{CI}: 1.89$ - 3.36), 2 - 5 years of medication $(\mathrm{AOR}=1.52,95 \% \mathrm{CI}: 1.35-1.88)$ were associated with dry eye in patients with glaucoma at KCMC. Conclusion: Dry eye is highly prevalent in glaucoma patients in our setting, and showed also the high prevalence of signs and symptoms. The use of timolol combined with other medication in our study was shown to be a strong predictor of having dry eye. To be exposed to two or more medication is strongly predictors of having dry eye.
\end{abstract}




\section{Keywords}

Dry Eye, Glaucoma

\section{Introduction}

Glaucoma is great public health concern and a leading cause of irreversible blindness worldwide. Global estimate indicates that 64.3 million people aged 40 to 80 years had glaucoma in 2019 and this number will increase to 76.0 million in 2020 and will reach 111.8 million in 2040 [1].

Dry eye is a common disease among glaucoma patients who are on topical medication for lowering intraocular pressure [2]; its prevalence with or without symptoms ranges from $5 \%$ to $50 \%$, and the prevalence based on symptoms alone is very higher around $75 \%$ [3]. The prevalence increases with age, affecting approximately $10 \%$ of those aged $30-60$ and $15 \%$ of adults over age 65 . Most epidemiologic studies have demonstrated a higher prevalence in women; it seems to occur with equal prevalence in all racial and ethnic group [4].

Dry eye is a multifactorial disease of the ocular surface characterized by a loss of homeostasis of the tear film, and accompanied by ocular symptoms, in which tear film instability, hyperosmolarity, ocular surface inflammation, damage, and neurosensory abnormalities play etiological roles [5]. This condition causes common ocular surface inflammatory disease that significantly affects quality of life, due to dysfunction of the lacrimal function unit, altering the tear composition and breaking ocular surface homeostasis, facilitating chronic inflammation and tissue damage [6].

Dry eye may be classified into two groups: dry eye with reduced tear production (aqueous-deficient) and dry eye with increased evaporation of the tear film (hyperevaporative) or a combination of the two [7].

Aqueous-deficient disorder affects around $10 \%$ of patients with dry eye. Around $80 \%$ patients of dry eye are due to combination of hyper evaporative/aqueous disorders [8].

Patients with tear dysfunction typically experience intermittent-to-constant eye irritation, photophobia, and blurred, foreign body sensation, stinging and fluctuating vision. These symptoms are often exacerbated by prolonged visual effort or a low-humidity environment, such as an airplane cabin. Chronic eye irritation may decrease quality of life in afflicted patients [8].

The risk factors associated with dry eye are well, including older people aged over 65 year, and women [9] [10]. Large incision of cataract surgery and penetrating keratoplasty, cigarette smoking, use of contact lens, collagen vascular disease, and irradiation were associated with dry eye [10]. Other factors for dry eyes include menopause, lasik and refractive excimer laser surgery, hematopoietic stem cell transplantation, vitamin A deficiency, hepatitis C, diuretics, beta-blockers, diabetes mellitus, infection like HIV/HTLV1, radiation therapy, low humidity environment, sarcoidosis, ovarian dysfunction, alcohol use, oral con- 
traceptives and pregnancy [8].

Ocular surface pain and discomfort is a major symptom of chronic dry eye and is frequently the primary reason patients seek an ophthalmologist. Clinically, there is disparity in the extent of tearing, corneal innervation, sensitivity, and pain among the patient population [8].

The diagnosis is made by questionnaire, functional test, diagnostic tests and examination of eyelid margins [11]

The differential diagnosis is made by exclusion with all kind of conjunctivitis, anterior blepharitis, dermodex, corneal and conjunctival abnormalities, filamentary and other keratitis, rheumatological conditions end graft versus host disease [5].

Treatment of dry eye disease involves a step ladder approach corresponding to disease severity and must take into account associated meibomian gland dysfunction, inflammation of the ocular surface, and/or associated systemic. That treatment is long term and may be slow to take effect disease [11].

The treatment includes lubricants, corticosteroid, cyclosporine and other anti-inflammatory agents, tetracyclines, macrolides, punctal plugs, bandage contact lens and scleral lens, eyelid hygiene [8].

This study aimed to determine the prevalence of dry eye; the risk factors associated with dry eye; and types and the severity of dry eye among glaucoma patients.

\section{Methods}

\subsection{Study Site}

This study was a hospital based prospective cross-sectional study at Kilimanjaro Christian Medical Center (KCMC), ophthalmology. KCMC Eye Department is a large specialized referral eye care provider located in Moshi Urban district. It has a catchment area of around 10 million people in Northern Tanzania. The eye department has a capacity of 36 beds, eight ophthalmologists (this includes subspecialists in surgical retina, cornea, glaucoma, paediatric ophthalmology and oculoplastic).

\subsection{Study Design}

The study population included all open angle (confirmed by gonioscopy) glaucoma patients with optic neuropathy, showing visual field defects on Humphrey Visual field analyzer, 18 years and above who attended the KCMC eye department on treatment for glaucoma during the study period (timolol and other medications like latanoprost, brimonidine, dorzalamide and selective laser trabeculoplasty). Exclusion criteria were patients with significant ocular surface disease (OSD) prior to commencing glaucoma treatment, including those treated with topical cyclosporine, steroids, or punctal plugs within the previous 3 months, history of other ocular inflammatory conditions (e.g. herpes simplex viral keratitis), presence of another ocular conditions affecting visual function (e.g. retinal 
pathology, non-glaucomatous optic neuropathy), were excluded.

Sample size was calculated by using the formula for cross section study [12], using data from study done in Nigeria [2], where the prevalence of dry eye was $68.9 \%$, and the minimal sample size was 324 and 325 patients were enrolled in this study.

\subsection{Data Collection}

The data was collected over a period of 8 months, from October 2018 to June of 2019, who was attended at KCMC eye clinic and fulfilled inclusion criteria, were identified and enrolled. All patients were screened using the ocular surface disease index (OSDI) paper based questionnaire, to assess symptoms and severity of OSD. To adapt the questionnaire to our setting we modified two questions as was done in the study carried out in Nigeria. We modified the question on "driving at night" to disturbances in vision noticed while walking at night or sitting in the front seat of the tricycle and the effect of the headlamp of an oncoming vehicle. "Computer/ATM" to effect of the phone screen on their eyes.

The demographic and history of medication like: medical treatment or surgical treatment, number of years, number of drops,

Clinical examination like: fluorescein breakup time, Ocular surface staining, Schirmer teste, meibomian gland were recorded.

\subsection{Data Analysis}

Data collected were coded and entered into computer using SPSS version 23 for statistical analysis.

Means (SD) and median (IQR) were used to summarize continuous variables. Proportion of dry eye was calculated and compared across different levels of categorical variables using Odds Ratio.

Odds Ratio was used to determine the magnitude of association between different factors and dry eye; and 95\% confidence interval and or a $p$ value $<0.05$ for statistical significance. The data were summarized and analyzed using the STATA software version 13 .

\subsection{Ethical Considerations}

Ethical approval number 2358 for this study was obtained from the KCMU College Ethical Committee prior to the commencement of this study. Permission to conduct this study was sought from the Director of KCMC hospital, Director of Post Graduate Studies and Head of Eye Department. All the patients have signed consent form. Confidentiality and privacy of study subjects were maintained and only the research team had access to it. IDs instead of names were used during analysis.

\section{Results}

This study included a total of 325 participants. The mean age (SD) of the study participants was 66.1 (11.8) years. Majority of the study participants 299 (92.0\%) 
were aged $\geq 50$ years, $184(56.6 \%)$ were males, 159 (48.9\%) had primary education. Three hundred and fourteen (96.6\%) were treated on medication, 216 (66.5\%) had 2 to 5 years of treatment, $208(64.0 \%)$ had no alternative treatment (prayers or traditional healers), 207 (63.7\%) were on single medication and most of the patients were treated with timolol eye drops 204 (62.8\%). An abnormal tear break up time was found in 283 patients (87.1\%) and 209 (64.3\%) had abnormal ocular surface staining, $184(56.6 \%)$ had normal Schirmer test; this is shown in Table 1.

\subsection{Prevalence of Dry Eye among the Glaucoma Patients at KCMC}

Based on OSDI score the prevalence of dry eye among patients with glaucoma at KCMC was $79.7 \%$ (259).

\subsection{Types of Dry Eye in Glaucoma Patients at KCMC}

Evaporative dry eye was found in $36.9 \%$ and aqueous deficiency in $43.4 \%$.

Majority of the study participants with evaporative dry eye (60.0\%) had mild meibomian gland deficiency (Figure 1).

\subsection{Factors Associated with Dry Eye in Glaucoma Patients at KCMC}

Factors such as use of two or more medication $(\mathrm{AOR}=2.55,95 \% \mathrm{CI}: 1.89$ 3.36), 2 - 5 years of medication ( $\mathrm{AOR}=1.52,95 \% \mathrm{CI}: 1.35$ - 1.88) were associated with dry eye in patients with glaucoma at KCMC. Other factors such as age, sex, education level, glaucoma management, alternative treatment, tear break up time, ocular surface staining, Schirmer test were not statistically significant associated with dry eye syndrome. This is shown in Table 2.

\subsection{The Severity of Dry Eye among Glaucoma Patients at KCMC}

Among those with dry eye majority of patients (61.9\%) had severe dry eye based on the ocular surface disease index, followed by mild dry eye. Table 3 shows more details.

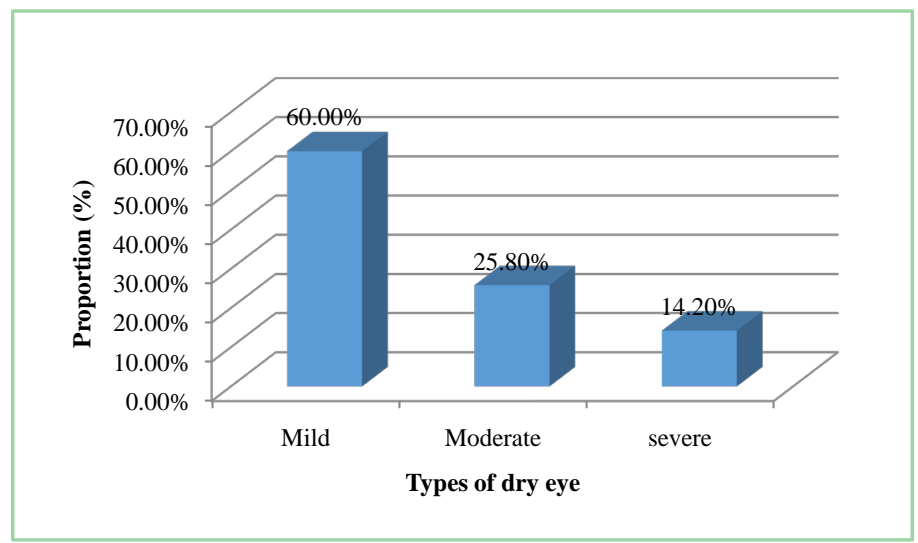

Figure 1. Meibomian gland dysfunction $(\mathrm{n}=120)$. 
Table 1. Characteristics of the study participants $(n=325)$.

\begin{tabular}{|c|c|c|c|}
\hline & \multicolumn{2}{|c|}{ OSDI Score } & \multirow[b]{3}{*}{ Total } \\
\hline & Normal & Dry eye & \\
\hline & n (\%) & n (\%) & \\
\hline Characteristics & $66(20.3)$ & $259(79.7)$ & n (\%) \\
\hline Age (years) (mean (SD)) & & & $66.1(11.8)$ \\
\hline \multicolumn{4}{|l|}{ Age (years) } \\
\hline$<50$ & $7(10.6)$ & $19(7.3)$ & $26(8.0)$ \\
\hline$\geq 50$ & $59(89.4)$ & $240(92.7)$ & $299(92.0)$ \\
\hline \multicolumn{4}{|l|}{ Sex } \\
\hline Male & $41(62.1)$ & $143(55.2)$ & $184(56.6)$ \\
\hline Female & $25(37.9)$ & $116(44.8)$ & $141(43.4)$ \\
\hline \multicolumn{4}{|l|}{ Education } \\
\hline None & $0(0.0)$ & $24(9.3)$ & $24(7.4)$ \\
\hline Primary & $27(40.9)$ & $132(50.9)$ & $159(48.9)$ \\
\hline Secondary & $18(27.3)$ & 49 (18.9) & $67(20.6)$ \\
\hline Tertiary & $21(31.8)$ & $54(20.9)$ & $75(23.1)$ \\
\hline \multicolumn{4}{|l|}{ Glaucoma treatment } \\
\hline Timolol & $40(60.6)$ & $164(63.3)$ & $204(62.8)$ \\
\hline Other medications & $26(39.4)$ & $95(36.7)$ & $121(37.2)$ \\
\hline \multicolumn{4}{|l|}{ Number of medication } \\
\hline Single & $40(60.6)$ & $167(64.5)$ & $207(63.7)$ \\
\hline Two or more medication & $26(39.4)$ & $92(35.5)$ & $118(36.3)$ \\
\hline \multicolumn{4}{|l|}{ Management } \\
\hline Medication & $62(93.9)$ & $252(97.3)$ & $314(96.6)$ \\
\hline Surgical & $4(6.1)$ & $7(2.7)$ & $11(3.4)$ \\
\hline \multicolumn{4}{|l|}{ Duration of treatment } \\
\hline$\leq 1$ & $10(15.1)$ & $35(13.5)$ & $45(13.9)$ \\
\hline 2 to 5 & $46(69.8)$ & $170(65.6)$ & $216(66.4)$ \\
\hline$>5$ & $10(15.1)$ & $54(20.9)$ & $64(19.7)$ \\
\hline \multicolumn{4}{|l|}{ Alternative treatment } \\
\hline No & $40(60.6)$ & $168(64.9)$ & $208(64.0)$ \\
\hline Traditional & $3(4.6)$ & $8(3.1)$ & $11(3.4)$ \\
\hline Prayers & $23(34.8)$ & $83(32.1)$ & $106(32.6)$ \\
\hline \multicolumn{4}{|l|}{ Tear break up time } \\
\hline Normal & $5(7.6)$ & $37(14.3)$ & $42(12.9)$ \\
\hline Abnormal & $61(92.4)$ & $222(85.7)$ & $283(87.1)$ \\
\hline \multicolumn{4}{|l|}{ Ocular surface staining } \\
\hline Normal & $24(36.4)$ & $92(35.5)$ & $116(35.7)$ \\
\hline Abnormal & $42(63.6)$ & $167(64.5)$ & $209(64.3)$ \\
\hline \multicolumn{4}{|l|}{ Schirmer test } \\
\hline Normal & $38(57.6)$ & $146(56.4)$ & $184(56.6)$ \\
\hline Dry eye & $28(42.4)$ & $113(43.6)$ & $141(43.4)$ \\
\hline
\end{tabular}


Table 2. Factors associated with dry eye in glaucoma patients $(n=325)$.

\begin{tabular}{|c|c|c|c|c|}
\hline \multirow[b]{2}{*}{ Factors } & \multicolumn{4}{|c|}{ Dry eye } \\
\hline & COR $(95 \% \mathrm{CI})$ & p-value & AOR $(95 \% \mathrm{CI})$ & p-value \\
\hline \multicolumn{5}{|l|}{ Age (years) } \\
\hline$<50$ & 1 & & 1 & \\
\hline$\geq 50$ & $1.49(0.60 ; 3.73)$ & 0.349 & $1.62(0.68 ; 3.54)$ & 0.277 \\
\hline \multicolumn{5}{|l|}{ Sex } \\
\hline Male & 1 & & 1 & \\
\hline Female & $1.33(0.76 ; 2.32)$ & 0.523 & $1.13(0.67 ; 1.82)$ & 0.688 \\
\hline \multicolumn{5}{|l|}{ Education } \\
\hline None & 1 & & 1 & \\
\hline Primary & $1.79(0.72 ; 4.41)$ & 0.209 & $2.13(0.82 ; 5.55)$ & 0.121 \\
\hline Secondary & $1.35(0.51 ; 3.59)$ & 0.548 & $1.73(0.57 ; 4.54)$ & 0.364 \\
\hline Tertiary & $1.41(0.54 ; 3.69)$ & 0.486 & $1.84(0.69 ; 5.49)$ & 0.209 \\
\hline \multicolumn{5}{|l|}{ Glaucoma treatment } \\
\hline Timolol & 1 & & 1 & \\
\hline Other medications & $0.89(0.51 ; 1.55)$ & 0.684 & $0.32(0.03 ; 3.88)$ & 0.368 \\
\hline \multicolumn{5}{|c|}{ Number of medication } \\
\hline Single & 1 & & 1 & \\
\hline$\geq 2$ Medication & $1.85(1.49 ; 2.48)$ & 0.004 & $2.55(1.89 ; 3.36)$ & 0.006 \\
\hline \multicolumn{5}{|l|}{ Management } \\
\hline Medication & 1 & & 1 & \\
\hline Surgical & $0.43(0.12 ; 1.52)$ & 0.181 & $2.16(0.83 ; 11.98)$ & 0.091 \\
\hline \multicolumn{5}{|l|}{ Duration of treatment } \\
\hline$\leq 1$ & 1 & & 1 & \\
\hline 2 to 5 & $1.56(1.49 ; 2.29)$ & 0.017 & $1.52(1.35 ; 1.88)$ & 0.005 \\
\hline$>5$ & $1.54(0.58 ; 4.09)$ & 0.383 & $0.69(0.31 ; 2.01)$ & 0.387 \\
\hline \multicolumn{5}{|l|}{ Alternative treatment } \\
\hline No & 1 & & 1 & \\
\hline Traditional & $0.63(0.16 ; 2.50)$ & 0.653 & $0.61(0.17 ; 2.23)$ & 0.451 \\
\hline Prayers & $0.86(0.48 ; 1.53)$ & 0.856 & $1.02(0.62 ; 1.99)$ & 0.934 \\
\hline \multicolumn{5}{|l|}{ Tear break up time } \\
\hline Normal & 1 & & 1 & \\
\hline Abnormal & $0.49(0.19 ; 1.31)$ & 0.154 & $1.08(0.53 ; 2.19)$ & 0.841 \\
\hline \multicolumn{5}{|c|}{ Ocular surface staining } \\
\hline Normal & 1 & & 1 & \\
\hline Abnormal & $1.04(0.59 ; 1.82)$ & 0.141 & $1.31(0.79 ; 2.17)$ & 0.289 \\
\hline \multicolumn{5}{|l|}{ Schirmer test } \\
\hline Normal & 1 & & 1 & \\
\hline Dry eye & $1.04(0.59 ; 1.82)$ & 0.899 & $1.31(0.79 ; 2.17)$ & 0.289 \\
\hline
\end{tabular}

COR stands for crude odds ratio; AOR stands for adjusted odds ratio. 
Table 3. Dry eye severity according to the ocular surface disease index $(n=231)$.

\begin{tabular}{cc}
\hline OSDI severity & $\mathbf{n} \%$ \\
\hline Mild & $51(22.1)$ \\
Moderate & $37(16.0)$ \\
Severe & $163(61.9)$ \\
\hline
\end{tabular}

\section{Discussion}

In this study, the prevalence of dry eye among glaucoma patients attended at KCMC eye department was $79.7 \%$. Evaporative dry eye was found in $36.9 \%$ and aqueous deficiency in $43.4 \%$. Dry eye was more prevalent among older aged participants ( $92.7 \%$ older than 50 years) and among males (55.2\%). KCMC being a referral hospital is expected to have higher prevalence of this condition, given that most of the patients are referred from other hospitals who might be already on treatment either with timolol only or with combination of medicines. Most of patients are on treatment for more than 2 years which may also increase their risk of having dry eyes. Another explanation is that our patients are mostly elderly (mean age 66.1 years) and is known that dry eye is more prevalent among older ages. The prevalence was similar in the study done by TF [2] and Craig [3] where the prevalence was $68.9 \%$ and $75 \%$ respectively. Our results differed from those obtained by Ruangvaravate [13] where the prevalence was $38.5 \%$, and by Fechtner [14] where the prevalence was $48.1 \%$. Rossi [15], found $42.1 \%$. In the study by Rossi, the Glaucoma Symptom Scale (GSS) instead of Ocular Surface Disease Index questionnaire was used which may account for the difference in the prevalence. The other studies were conducted in other geographic areas with different climatic conditions which may also affect the prevalence of dry eye. Also though not stated in the articles, in USA and other developed countries patients are more likely to have access to preservative free medications thus will have less dry eye symptoms.

In this study we found that the use of two or more medication ( $\mathrm{AOR}=2.55$, $95 \%$ CI: 1.89 - 3.36), 2 - 5 years of medication $(\mathrm{AOR}=1.52$, 95\% CI: $1.35-1.88)$, was associated with dry eye in patients with glaucoma. Numerous reports have shown that preservative containing eye drops increase symptoms of dry eyes mainly by the adverse effects on the conjunctiva and cornea, by induction of inflammation, reduction in corneal barrier and destabilization of the corneal tear film [16]. The same results were obtained in study done by Camp [17] which found that the number of drops was associated with severity of dry eye $p=0.03$. Also our findings were similar to results obtained by $\mathrm{Tf}$ [2], where they found that increasing number of drops was associated with dry eye symptoms $\mathrm{p} \leq 001$. All the medications used in treating glaucoma contain preservatives, mainly Benzalkonium Chloride. According to the literature by Messmer [8], age above 50 years and famale gender were also high risk factors for dry eye, but in our study they were not statistically significant. Those that were aged 50 years and above had $49 \%$ higher odds of dry eye than patients less than 50 years; though 
not statistically significant, the risk was higher in older patients. Gender was not associated with dry eye in our study, but we had more male than female participants. Many studies have shown a poor association between dry eye symptoms and clinical features on examination as is the case in our study, where based on the ocular surface staining $64 \%$ of patients had dry eye and based on the Schirmer test $43.6 \%$ had dry eye. This was different from $79.7 \%$ obtained based on symptoms using OSDI questionnaire. According to a systematic review by Bartlett [18] where 33 articles on the association between signs and symptoms of dry were reviewed; they found that only $24 \%$ had statistically significant association showing a correlation of between -0.4 and 0.4 , indicating low-to-moderate correlation. Rossi [15] found that gender independent and punctate keratitis was associated with age $(\mathrm{p}$ value $=0.01)$ and with the number of instillation $\mathrm{p}=$ 0.007 . In this survey the study design was different, it was prospective observational study and they enrolled also patient with ocular hypertension.

\section{Limitations}

Also, KCMC is a referral center, implying that most of the patients will have poorly controlled glaucoma and will be more likely to be on multiple medications and to have symptoms of dry eye.

\section{Conclusions}

Based on our study, we concluded the following:

That dry eye is a common problem and highly prevalent in glaucoma patients in our setting, and showed also the high prevalence of signs and symptoms. The use of timolol between 2 to 5 years in our study was shown to be a strong predictor of having dry eye. To be exposed to two or more medication was strongly predictors of having dry eye.

\section{Acknowledgements}

We thank Dr Heiko Philippin, Dr Lydia Fukunag, Dr Abrahamo Matova, Sia Mbishi, Lydia Masika for their contribution on this study, to all patients who participated on this study. Also I extend my gratitude to my sponsor Light for the World for preventing blindness in Mozambique.

\section{Conflicts of Interest}

The authors declare that they have no conflict of interest.

\section{References}

[1] Tham, Y.C., Li, X., Wong, T.Y., Quigley, H.A., Aung, T. and Cheng, C.Y. (2014) Global Prevalence of Glaucoma and Projections of Glaucoma Burden through 2040. A Systematic Review and Meta-Analysis. Ophthalmology, 121, 2081-2090. https://doi.org/10.1016/j.ophtha.2014.05.013

[2] Tf, S., Fasina, O., Ashaye, A., Bekibele, C. and Olawoye, O. (2015) Ocular Surface Disease among Glaucoma Patients in Ibadan, South-West Nigeria. Journal of Oph- 
thalmology of Eastern Central and Southern Africa, 19, 34-38.

[3] Craig, J.P., et al. (2017) TFOS DEWS II Report Executive Summary. The Ocular Surface, 15, 802-812. https://doi.org/10.1016/j.jtos.2017.08.003

[4] Foulks, G.N. (2003) Cornea and External Disease. Ophthalmology Clinics of North America, 16, 44-58. https://doi.org/10.1016/S0896-1549(02)00109-8

[5] Craig, J.P., et al. (2017) TFOS DEWS II Definition and Classification Report. Ocular Surface, 15, 276-283. https://doi.org/10.1016/j.jtos.2017.05.008

[6] Stern, M.E., Schaumburg, C.S. and Pflugfelder, S.C. (2013) Dry Eye as a Mucosal Autoimmune Disease. International Reviews of Immunology, 32, 19-41. https://doi.org/10.3109/08830185.2012.748052

[7] Osae, A.E., et al. (2017) Epidemiology of Dry Eye Disease in Africa: The Sparse Information, Gaps and Opportunities. Ocular Surface, 15, 159-168. https://doi.org/10.1016/j.jtos.2017.01.001

[8] Messmer, E.M. (2015) The Pathophysiology, Diagnosis, and Treatment of Dry Eye Disease. Deutsches Ärzteblatt International, 112, 71-81. https://doi.org/10.3238/arztebl.2015.0071

[9] Shanti, Y., Shehada, R., Bakkar, M.M. and Qaddumi, J. (2020) Prevalence and Associated Risk Factors of Dry Eye Disease in 16 Northern West Bank Towns in Palestine: A Cross-Sectional Study. BMC Ophthalmology, 20, 26. https://doi.org/10.1186/s12886-019-1290-Z

[10] Outcomes, M. (2019) Characteristics and Risk Factors Associated with Diagnosed and Undiagnosed Symptomatic Dry Eye Using a Smartphone Application. JAMA Ophthalmology, 138, 58-68. https://doi.org/10.1001/jamaophthalmol.2019.4815

[11] Paulsen, A.J., et al. (2014) Dry Eye in the Beaver Dam Offspring Study: Prevalence, Risk Factors, and Health-Related Quality of Life. American Journal of Ophthalmology, 157, 799-806. https://doi.org/10.1016/j.ajo.2013.12.023

[12] Charan, J. and Biswas, T. (2013) How to Calculate Sample Size for Different Study Designs in Medical Research? Indian Journal of Psychological Medicine, 35, 121. https://doi.org/10.4103/0253-7176.116232

[13] Ruangvaravate, N., Prabhasawat, P., Vachirasakchai, V. and Tantimala, R. (2018) High Prevalence of Ocular Surface Disease among Glaucoma Patients in Thailand. Journal of Ocular Pharmacology and Therapeutics, 34, 387-394.

[14] Fechtner, R.D., Godfrey, D.G., Budenz, D., Stewart, J.A., Stewart, W.C. and Jasek, M.C. (2010) Prevalence of Ocular Surface Complaints in Patients with Laucoma Using Topical Intraocular Pressure-Lowering Medications. Cornea, 29, 618-621. https://doi.org/10.1097/ICO.0b013e3181c325b2

[15] Rossi, G.C.M., Pasinetti, G.M., Scudeller, L. and Bianchi, P.E. (2013) Ocular Surface Disease and Glaucoma: How to Evaluate Impact on Quality of Life. Journal of Ocular Pharmacology and Therapeutics, 29, 390-394.

https://doi.org/10.1089/jop.2011.0159

[16] Yee, R.W. (2007) The Effect of Drop Vehicle on the Efficacy and Side Effects of Topical Glaucoma Therapy: A Review. Current Opinion in Ophthalmology, 18, 134-139. https://doi.org/10.1097/ICU.0b013e328089f1c8

[17] Camp, A., et al. (2015) Dry Eye Specific Quality of Life in Veterans Using Glaucoma Drops. Contact Lens \& Anterior Eye, 38, 220-225. https://doi.org/10.1016/j.clae.2015.02.001

[18] Bartlett, J.D. and Keith, M.S. (2015) Associations between Signs and Symptoms of Dry Eye Disease: A Systematic Review. Clinical Ophthalmology, 9, 1719-1730. https://doi.org/10.2147/OPTH.S89700 